\title{
Sphincter-Preserving Therapy for Treating a Chronic Anal Fissure: Long-term Outcomes
}

\author{
Ridzuan Farouk \\ Department of Surgery, National University Hospital, Singapore
}

Purpose: To estimate the risk of recurrent fissure in ano after sphincter preserving treatments.

Methods: A retrospective case note review, combined with a telephone survey was conducted for all patients treated for a chronic anal fissure between 1998 and 2008.

Results: Six hundred and twelve patients (303 women: mean age, 39 years; range, 16-86 years) were treated for chronic anal fissure between 1998 and 2008. Topical diltiazem 2\% was initially prescribed for 8 weeks. The fissure did not heal in 141 patients. These patients (61 women: mean age, 30 years; range, 15-86 years) were treated with 100 IU botulinum A toxin (Botox) injection combined with a fissurectomy under general anaesthesia. Thirty eight patients suffered a recurrence of their fissure within two years. Thirty-four healed with further medical or sphincter conserving surgical therapy while four required a lateral internal sphincterotomy.

Conclusion: The vast majority of patients with chronic anal fissure can be treated with sphincter conserving treatments. This may require several interventions before healing can be achieved. Assessment for recurrence after 'conservative' treatments requires a minimum of two-year follow-up.

\section{Keyword: Fissure in ano}

\section{INTRODUCTION}

A reversible chemical sphincterotomy using topical glyceryl trinitrate (GTN) was described as a potential medical therapy for chronic anal fissure in 1991 [1]. Application of GTN is associated with a significant reduction in resting anal pressure and a reduction in pain scores [2]. Long-term healing can be achieved in 59\% of patients treated in this way [2]. Significant side-effects of headache and poor fissure healing rates have discouraged some from using a chemical sphincterotomy as a treatment option for chronic anal fissure [3]. A lateral sphincterotomy results in superior healing rates [4] and remains the gold standard treatment for

Received: September 3, 2013 - Accepted: November 24, 2013

Correspondence to: Ridzuan Farouk, LLB (Hons) MCh FRCS

Department of Surgery, National University Hospital, Tower Block \#8,

1 E Kent Ridge Road, Singapore 119228, Singapore

Tel: +65-6772-4235, Fax: +65-6777-8206

E-mail: ridzuan_farouk@nuhs.edu.sg

(c) 2014 The Korean Society of Coloproctology

This is an open-access article distributed under the terms of the Creative Commons Attribution NonCommercial License (http://creativecommons.org/licenses/by-nc/3.0) which permits unrestricted non-

commercial use, distribution, and reproduction in any medium, provided the original work is properly cited. chronic anal fissure [5]. The risk of incontinence after a lateral sphincterotomy is small [5], but its role in treating women with a significant obstetric history, older patients, and those who have undergone previous anal surgery has spurred the development of alternative sphincter-sparing treatments for patients with chronic anal fissure.

Anal advancement flaps [6] and botulinum A toxin (Botox) injection combined with a fissurectomy [7] represent alternative surgical approaches that preserve anal sphincter integrity. The significant side-effect profile of GTN has resulted in alternative pharmacological forms of a chemical sphincterotomy including Botox injection and topical diltiazem application $[8,9]$. This paper reports our experience with sphincter-preserving treatments in consecutive patients presenting with chronic anal fissure. Longterm outcomes are reported along with the details of any salvage treatments delivered.

\section{METHODS}

Chronic anal fissure is defined as a visible split in the midline of the anoderm with symptoms of anal pain and/or bleeding present for a minimum of six weeks. Patients with Crohn's disease, radia- 
tion proctitis or slow transit constipation were excluded from the study.

All patients were treated with topical diltiazem $2 \%$ ointment applied twice daily for eight weeks. Additional treatment with a high-fiber diet and a stool softener was encouraged in patients who did not suffer from diarrhea. Patients whose fissure had healed were discharged with advice to continue with a high-fiber diet and a stool softener for a minimum of three months. Those with a persistent fissure were prescribed with a second course of diltiazem for an additional eight weeks. A persistent fissure despite sixteen weeks of topical diltiazem resulted in a fissurectomy and injection of $100 \mathrm{IU}$ of botulinum A toxin (Botox). Patients whose fissure did not heal after a fissurectomy and Botox injection were offered a choice of GTN ointment, a second Botox injection with fissurectomy, an anal advancement flap, or lateral sphincterotomy.

Late patient outcomes were determined by telephone interviews two and five years after surgery. When patients could not be contacted, their family doctors were contacted and their hospital records were checked to determine whether any additional treatments had later become necessary. These processes were repeated again in 2007, 2010, and 2013 to ensure completeness of data collection.

\section{RESULTS}

Between 1998 and 2008, 612 patients (303 women and 309 men) were treated for a chronic anal fissure by using topical diltiazem $2 \%$ ointment four hospitals (Royal Berkshire Hospital, West Berkshire Community Hospital, Dunedin Hospital, and Berkshire Independent Hospital, Reading, UK). The median age of the patients treated was 39 years (range, 15-86 years). Thirty patients (5\%) developed a contact dermatitis as a result of using the cream, and ninety-two patients (15\%) complained of pruritus ani during or shortly after completion of their treatment.

One hundred forty-one patients (23\%) either did not heal with topical diltiazem ointment or relapsed after stopping treatment. The median age of these patients was 30 years (range, 16-86 years). All underwent a fissurectomy and had 100-IU Botox injected, divided in 50-IU aliquots, into the intersphincteric space at the three and nine oclock positions with the patient in the lithotomy position under general anesthesia. Eleven patients (8\%) reported transient urgency for defecation lasting up to twelve weeks after this procedure.

Thirty-eight of these one hundred and forty-one patients (26\%) did not heal immediately after their fissurectomy \& Botox procedure (12 patients) or suffered a recurrence within two years of their surgery (25 patients). Nine healed with topical GTN cream, 20 underwent a second fissurectomy and Botox injection, five were treated with an anal advancement flap and four patients underwent lateral internal sphincterotomy. Two patients did not respond to these repeat treatments and were referred elsewhere for further treatment.

One patient who had undergone a lateral internal sphincterotomy reported permanent fecal urgency at the two- and the fiveyear follow-ups.

\section{DISCUSSION}

The majority of patients presenting with chronic anal fissure have evidence of reduced anodermal blood flow [10], high resting anal pressure $[2,11]$ and ultraslow waves [11] suggestive of internal anal sphincter spasms. Lateral internal sphincterotomy represents the gold standard of treatment [5] against which new treatments should be compared. Early recurrence after sphincter-conserving therapy is common, and this research would suggest that a minimum of two-year follow-up is required to determine the efficacy of alternative treatments.

Endoanal ultrasound combined with anal manometry has established a cohort of patients with an anal fissure at increased risk of fecal incontinence following a lateral sphincterotomy. These include women with an obstetric history [12], patients who have undergone previous anal surgical procedures [13, 14], and older patients with a short anal sphincter [15].

Topical diltiazem $2 \%$ ointment was used as it appears to be at least equally efficacious as GTN but with fewer side-effects. A high-fiber diet and the use of a stool softener during treatment and for a period afterwards has in all likelihood contributed to improved healing rates and minimized long-term recurrence. The primary side-effects of diltiazem $2 \%$ ointment appear to be perianal dermatitis and pruritus ani.

Failure of treatment with topical diltiazem resulted in a fissurectomy and Botox injection. The fissurectomy, performed with a curette, converts the chronic fissure into an acute wound. The mechanism of action of the Botox injection remains unclear, but it may improve anodermal blood flow either by chemical relaxation of the anal sphincter and/or vasodilation.

The dose of Botox used in this study is higher than that published in earlier series but has not translated into high rates of fecal incontinence. The 100 units are diluted into $2 \mathrm{~mL}$. of saline allowing $50 \mathrm{IU}$ aliquots of Botox to be injected into the intersphincteric space. Whether there is any advantage of this injection site over others remains to be resolved but the 3 and 9 oclock positions using an anal speculum to demonstrate the intersphincteric space is surgically convenient. The ideal dose of Botox required is also unresolved with previous reports varying the amount of Botox used varying between 12 and $50 \mathrm{IU}$ per injection.

Initial success after treatment of an anal fissure, as measured by resolution of pain and healing of the fissure, is tempered by recurrent symptoms, and treatment efficacy should only be judged with adequate follow-up. Twenty-five of our patients who initially responded to their Botox injection and fissurectomy relapsed at a later time. A minimum two-year follow-up appears necessary to adequately determine treatment success. 
A second cycle of topical cream or a fissurectomy \& Botox injection can sometimes avoid a subsequent sphincterotomy. Patient motivation, counseling of risks involved with treatment options available, and the ability to deliver a package of treatment options, including undertaking an anal advancement flaps, determine which steps are available to minimize the need for sphincter division, thereby keeping the risks of incontinence to a minimum [16].

In conclusion, conservative therapy for a chronic anal fissure includes medical and surgical techniques. A stepwise approach to treatment that incorporates medical and surgical treatments is time consuming and requires patient counseling, but ultimately such an approach can successfully preserve the integrity of the anal sphincter muscle for the majority of patients. Recurrences are common, so long-term follow-ups of patients who underwent conservative therapies are required to fully assess the efficacy of treatment.

\section{CONFLICT OF INTEREST}

No potential conflict of interest relevant to this article was reported.

\section{REFERENCES}

1. Loder PB, Kamm MA, Nicholls RJ, Phillips RK. 'Reversible chemical sphincterotomy' by local application of glyceryl trinitrate. Br J Surg 1994;81:1386-9.

2. Kennedy ML, Sowter S, Nguyen H, Lubowski DZ. Glyceryl trinitrate ointment for the treatment of chronic anal fissure: results of a placebo-controlled trial and long-term follow-up. Dis Colon Rectum 1999;42:1000-6.

3. Altomare DF, Rinaldi M, Milito G, Arcana F, Spinelli F, Nardelli N, et al. Glyceryl trinitrate for chronic anal fissure--healing or headache? Results of a multicenter, randomized, placebo-controled, double-blind trial. Dis Colon Rectum 2000;43:174-9.

4. Libertiny G, Knight JS, Farouk R. Randomised trial of topical $0.2 \%$ glyceryl trinitrate and lateral internal sphincterotomy for the treatment of patients with chronic anal fissure: long-term followup. Eur J Surg 2002;168:418-21.
5. Nelson RL. Operative procedures for fissure in ano. Cochrane Database Syst Rev 2010;(1):CD002199.

6. Nyam DC, Wilson RG, Stewart KJ, Farouk R, Bartolo DC. Island advancement flaps in the management of anal fissures. Br J Surg 1995;82:326-8.

7. Scholz T, Hetzer FH, Dindo D, Demartines N, Clavien PA, Hahnloser D. Long-term follow-up after combined fissurectomy and Botox injection for chronic anal fissures. Int J Colorectal Dis 2007;22:1077-81.

8. Knight JS, Birks M, Farouk R. Topical diltiazem ointment in the treatment of chronic anal fissure. Br J Surg 2001;88:553-6.

9. Samim M, Twigt B, Stoker L, Pronk A. Topical diltiazem cream versus botulinum toxin a for the treatment of chronic anal fissure: a double-blind randomized clinical trial. Ann Surg 2012;255:1822.

10. Schouten WR, Briel JW, Auwerda JJ, De Graaf EJ. Ischaemic nature of anal fissure. Br J Surg 1996;83:63-5.

11. Farouk R, Duthie GS, MacGregor AB, Bartolo DC. Sustained internal sphincter hypertonia in patients with chronic anal fissure. Dis Colon Rectum 1994;37:424-9.

12. Corby H, Donnelly VS, O'Herlihy C, O'Connell PR. Anal canal pressures are low in women with postpartum anal fissure. $\mathrm{Br} \mathrm{J}$ Surg 1997;84:86-8.

13. Farouk R, Bartolo DC. The use of endoluminal ultrasound in the assessment of patients with faecal incontinence. J R Coll Surg Edinb 1994;39:312-8.

14. Tjandra JJ, Han WR, Ooi BS, Nagesh A, Thorne M. Faecal incontinence after lateral internal sphincterotomy is often associated with coexisting occult sphincter defects: a study using endoanal ultrasonography. ANZ J Surg 2001;71:598-602.

15. Frudinger A, Halligan S, Bartram CI, Price AB, Kamm MA, Winter R. Female anal sphincter: age-related differences in asymptomatic volunteers with high-frequency endoanal US. Radiology 2002; 224:417-23.

16. Hancke E, Rikas E, Suchan K, Volke K. Dermal flap coverage for chronic anal fissure: lower incidence of anal incontinence compared to lateral internal sphincterotomy after long-term followup. Dis Colon Rectum 2010;53:1563-8. 